\title{
Identification of snoRNA SNORA71A as a Novel Biomarker in Prognosis of Hepatocellular Carcinoma
}

\author{
Yuan Ding, ${ }^{1,2,3,4,5}$ Zhongquan Sun,, ${ }^{1,2,3,4,5}$ Sitong Zhang, ${ }^{1,2,3,4,5}$ Liuzhi Zhou ${ }^{1,1,2,3,4,5}$ \\ Qianhui Xu, ${ }^{1,2,3,4,5}$ Dongkai Zhou, ${ }^{1,2,3,4,5}$ Yanjie Li, ${ }^{1,2,3,4,5}$ Xin Han, ${ }^{1,2,3,4,5}$ Hao Xu, 1,2,3,4,5 \\ Yang Bai, ${ }^{1,2,3,4,5}$ Chang Xu, ${ }^{1,2,3,4,5}$ Hao Ding, ${ }^{1,2,3,4,5}$ Yao Ge, ${ }^{2,3,4,5}$ and Weilin Wang $\mathbb{D}^{1,2,3,4,5}$ \\ ${ }^{1}$ Department of Hepatobiliary and Pancreatic Surgery, The Second Affiliated Hospital, Zhejiang University School of Medicine, \\ Hangzhou 310009, China \\ ${ }^{2}$ Key Laboratory of Precision Diagnosis and Treatment for Hepatobiliary and Pancreatic Tumor of Zhejiang Province, \\ Hangzhou 310009, China \\ ${ }^{3}$ Research Center of Diagnosis and Treatment Technology for Hepatocellular Carcinoma of Zhejiang Province, \\ Hangzhou 310009, China \\ ${ }^{4}$ Clinical Medicine Innovation Center of Precision Diagnosis and Treatment for Hepatobiliary and Pancreatic Disease of \\ Zhejiang University, Hangzhou 310009, China \\ ${ }^{5}$ Clinical Research Center of Hepatobiliary and Pancreatic Diseases of Zhejiang Province, Hangzhou 310009, China
}

Correspondence should be addressed to Weilin Wang; wam@zju.edu.cn

Received 3 April 2020; Revised 2 August 2020; Accepted 10 September 2020; Published 27 September 2020

Academic Editor: Stamatios E. Theocharis

Copyright (c) 2020 Yuan Ding et al. This is an open access article distributed under the Creative Commons Attribution License, which permits unrestricted use, distribution, and reproduction in any medium, provided the original work is properly cited.

\begin{abstract}
Background. Small nucleolar RNAs (snoRNAs) have been proved to play important roles in various cellular physiological process. Recently, dysregulation of snoRNA SNORA71A has been found involved in tumorigenesis of various malignant cancers. However, the emerging effects of SNORA71A in hepatocellular carcinoma (HCC) remain largely unclear. In this study, we aimed to explore the SNORA71A expression and its underlying significance in HCC. Methods. Expression of SNORA71A in cell lines and clinical specimens was measured by quantitative real-time PCR. Then, all enrolled HCC patients were divided into low and high SNORA71A expression subgroups and then they were compared in the aspects of clinical features as well as survival outcome by respective statistical analysis methods. Results. SNORA71A was significantly downexpressed in SK-HEP-1 $(P=0.001)$, Huh-7 $(P<0.001)$, Hep3B $(P<0.001)$, and clinical HCC specimens $(P=0.006)$. Comparing the clinical features between SNORA71A expression subgroups, it showed that low SNORA71A expression was significantly associated with large tumor diameter, multiple lesions, capsular invasion, bad tumor differentiation, and TNM stage $(P<0.05)$. Furthermore, it was found that HCC patients with lower SNORA71A expression had higher risk in postoperative tumor relapse (median time: 9.5 vs. 35.2 months; low vs. high; $P<0.001$ ) and poor overall survival (median time: 36.8 vs. 52.9 months; low vs. high; $P<0.001$ ). Besides, SNORA71A expression served as independent risk factors for tumor-free $(\mathrm{HR}=0.450 ; 95 \% \mathrm{CI}[0.263-0.770] ; P=0.004)$ and long-term survival ( $\mathrm{HR}=0.289 ; 95 \% \mathrm{CI}[0.127-0.657] ; P=0.003)$. Conclusions. Our study for the first time demonstrated that downregulation of SNORA71A could serve as a novel biomarker for clinical assessment and prognostic prediction of HCC patients.
\end{abstract}

\section{Introduction}

Hepatocellular carcinoma (HCC) is the fifth most common cancer and causes the fourth most cancer-related deaths in the world [1]. There are a series of risk factors to induce the tumorigenesis of HCC, mainly including chronic hepatitis virus infection, fatty diabetes, excessive alcohol intake, and nonalcoholic fatty liver disease [2]. Despite great achievements in the treatment of hepatocellular carcinoma with respect to liver resection and chemotherapy, the survival outcomes of HCC patients remain dissatisfactory for its high incidence of relapse, metastasis, and mortality [3]. Even as the most commonly and important means for HCC diagnosis, serum AFP monitoring is not sufficient to predict the 
postoperative survival for HCC patients effectively [4]. Therefore, it is vital and significant to identify a novel reliable clinical marker for HCC prognostication.

Small nucleolar RNA (snoRNA) is a type of noncoding RNA widely found in the nucleoli of eukaryotic cells [5]. It has a length of 0-300 nt and has conserved structural elements. Although these snoRNAs were once considered with single function and limited effects in pre-rRNA processing, recent accumulating evidence showed that some snoRNAs also participated in various cellular physiological processes, including cell proliferation, differentiation, epigenetic, regulation. And some dysregulation of snoRNA was proved to serve as tumor suppressor genes or oncogenes in various cancers [6-9]. A review from Han et al. clearly stated that SNHG1, SNHG6, SNHG15, SNHG16, and SNHG20 can play varied roles in HCC through different regulatory mechanisms. These SNHGs can promote and inhibit tumorigenesis [10]. For instance, snoRNA SNHG1 promoted tumorigenesis and progression of colorectal cancer via negative regulation of miR-137 [11]. Besides, snoRNA SNORA18 acted as a tumor suppressor gene in hepatocellular carcinoma [12].

SNORA71A, encoded by the third intron of snoRNA host gene 17 (SNHG17) cloning, commonly guided the pseudouridine of U406 in 18S rRNA [13]. In recent, more and more studies reported that SNORA71A was dysregulated in various types of cancers, such as lung cancer [14] and breast cancer [15], and played crucial roles in tumor progression. However, the emerging effects and potential mechanisms of SNORA71A in hepatocellular carcinoma remain largely unclear. In this study, we aimed to identify the SNORA71A expression features in HCC and its underlying clinical significance.

\section{Materials and Methods}

2.1. Patients and Tissue Samples. To reduce the potential confounding factors, none of the enrolled patients received any preoperative radiotherapy, chemotherapy, or endocrine therapy. All enrolled patients were diagnosed with HCC by histopathological examination. All patients involved in the study were written informed consent and approval. This study was approved by the Institutional Ethics Committee of the First Affiliated Hospital of Zhejiang University (Hangzhou, China).

HCC tissues and adjacent liver tissues were received from 132 consecutive HCC patients, who underwent liver resection between January 2013 and December 2014. HCC tissue specimens underwent 2 pathological examinations by pathologists for TNM stage. Tissues were immediately snap frozen into liquid nitrogen postoperation and then stored at $-80^{\circ} \mathrm{C}$ for RNA isolation. Patient data was collected from the database of the hospital electronic system, including individual information, liver function indexes, alpha fetal protein value, HBV infection, lesion features, pathological differentiation, and TNM classification.

2.2. Follow-Up of Tumor-Free and Long-Term Survival. After hepatectomy, all patients were followed up for 5 years. After the initial surgery, the time terminal of tumor-free survival

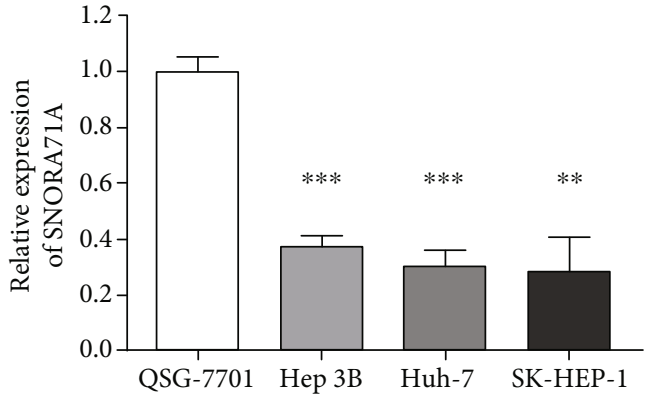

Figure 1: Comparison of SNORA71A expression in cell lines. Compared to normal liver cell line QSG-7701, SNORA71A was significantly suppressed in HCC cell lines (SK-HEP-1, $P=0.001$; Huh-7, $P<0.001$; Hep3B, $P<0.001) .{ }^{* *} P<0.01$ and ${ }^{* * *} P<0.001$.

included the recurrence of HCC, HCC distant metastasis, or death from any cause without cancer-related events, while long-term survival was calculated until death or the last follow-up time in 5 years. Death of patients was ascertained by the family members and the review of public hospital records. All staff who collected all the following-up data of enrolled patients were blind to participant status.

2.3. Cell Culture. Human normal hepatocyte cell line (QSG7701) and HCC cell lines (SK-HEP-1, Huh-7, and Hep3B) obtained from the laboratory were maintained in the DMEM medium (BI, Israel) with 10\% FBS (BI, Israel) as well as $100 \mathrm{U} / \mathrm{ml}$ penicillin and $100 \mathrm{mg} / \mathrm{ml}$ streptomycin (Sangon, China) at $37^{\circ} \mathrm{C}$ in a humidified incubator (Thermo Fisher, USA) containing $5 \% \mathrm{CO}_{2}$.

2.4. RNA Extraction and Quantitative Real-Time PCR. Total RNA from cell lines and tissue specimens was extracted using Trizol reagent (Invitrogen, USA) according to the manufacturer's instructions. RNA was reversely transcribed into cDNA using cDNA Reverse Transcription Kit (Vazyme, Nanjing, China). To determine the expression level of SNORA71A, cDNA sample was tested with quantitative real-time polymerase chain reaction (RT-PCR) (Roche, Basel, Switzerland). All samples were tested in triplicate. The expression of glyceraldehyde-3-phosphate dehydrogenase (GAPDH) was used as an endogenous control, and the relative expression of SNORA71A was calculated by comparative $\mathrm{Ct}$ method formula $2^{-\Delta \Delta \mathrm{Ct}}$. The sequences of all PCR primers used were as follows $\left(5^{\prime}-3^{\prime}\right)$ : GAPDH: CAGGAG GCATTGCTGATGAT (forward), GAAGGCTGGGGCTC ATTT (reverse); SNORA71A: AGGTCATTGATAGTGCA GGGAG (forward), GGTTCGGATGGGATAGGGT (reverse).

2.5. Statistical Analysis. All statistical analyses were performed using the SPSS (Statistical Package for the Social Sciences) 19.0 (SPSS, Chicago, IL). The differences between two independent groups were analyzed using Student's $t$-test. The correlations between SNORA71A expression and different clinical characteristics were performed with chi-squared test. The differences of tumor-free and long-term survival in different subgroups were assessed by Kaplan-Meier survival plots and log-rank tests. Associations between 


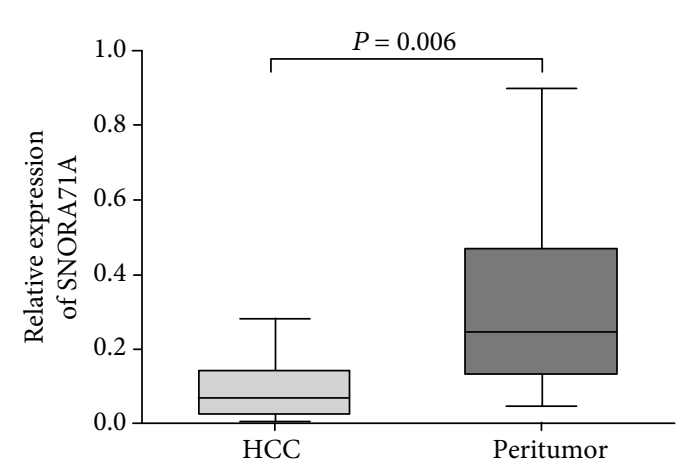

(a)

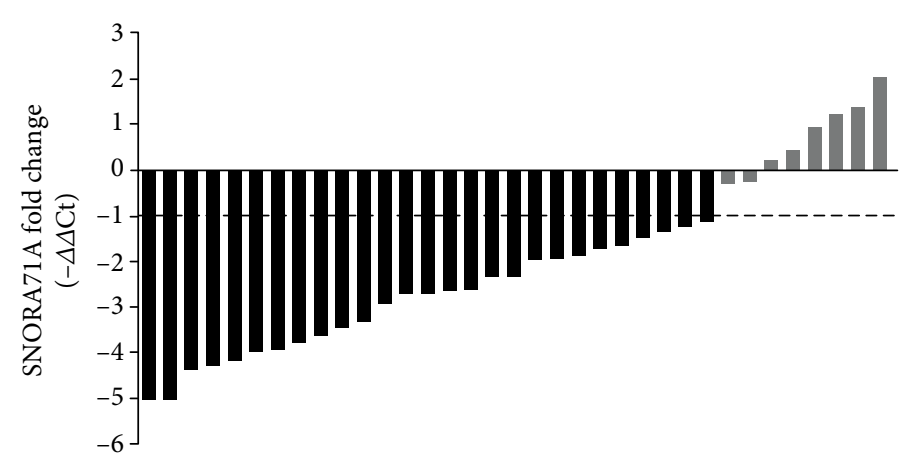

(b)

FIGURE 2: Expression patterns of SNORA71A in HCC tissue specimens. (a) Compared to corresponding adjacent liver tissues, SNORA71A expression was significantly lower in HCC tissues $(P<0.01)$. (b) Waterfall plot showed that SNORA71A was downregulated by at least twofold in $77.2 \%$ of paired HCC tissues. $\triangle \triangle \mathrm{Ct}=(\mathrm{Ct}$ SNORA71A $-\mathrm{Ct}$ GAPDH $)$ of HCC lesions $-(\mathrm{Ct}$ SNORA71A $-\mathrm{Ct}$ GAPDH $)$ of adjacent liver tissue.

HCC prognosis and clinical parameters were first performed with univariate analysis and then gradually analyzed using multiple logistic regression for those significantly differing factors. All data were expressed with mean \pm standard deviation (SD), and $P<0.05$ was recognized as statistically significant difference.

\section{Results}

3.1. The Expression Levels of SNORA71A in HCC. To determine whether SNORA71A was differentially expressed in HCC, firstly human normal hepatocyte (QSG-7701) and HCC cell lines (SK-HEP-1, Huh-7, and Hep3B) were used to analyze SNORA71A expression by quantitative RT-PCR. As shown in Figure 1, the SNORA71A expression in SK-HEP-1 $(P=0.001)$, Huh-7 $(P<0.001)$, and Hep3B $(P<0.001)$ were remarkably lower than QSG-7701, respectively. Then, as shown in Figure 2, the similar results were also found in clinical tissues' examination that SNORA71A expression was mostly downexpressed in HCC tissues compared with adjacent liver tissues $(P=0.006)$. Therefore, these results suggested that SNORA71A might serve as a tumor suppressive role in HCC.

\subsection{Relationships of SNORA71A Expression with} Clinicopathologic Characteristics of HCC Patients. According to individual SNORA71A expression, all 132 HCC patients were divided into high and low expression subgroups. The relationships of SNORA71A expression with clinicopathologic characteristics of HCC patients were statistically analyzed. In Table 1, low SNORA71A expression was significantly associated with large tumor diameter $(P=0.016)$, multiple lesions $(P=0.017)$, capsular invasion $(P=0.015)$, bad tumor differentiation $(P=0.027)$, and TNM stage $(P=0.004)$. However, there was no statistical significance between SNORA71A expression with other clinicopathologic characteristics, such as hepatitis B, AFP, or cirrhosis. HCC Patients. To identify whether SNORA71A expression could serve as a novel biomarker to predict prognosis of HCC patients, the tumor-free and long-term survival data of two SNORD31 expression subgroups were compared using Kaplan-Meier method. According to the results from Figures 3 and 4, we found that HCC patients with high SNORA71A expression tended to present with lower tumor recurrence rate after hepatectomy and better overall survival condition. Between the subgroups with different SNORA71A expression levels, the median tumor-free survival time $(9.5$ vs. 35.2 months; low vs. high; $P<0.001)$ and median longterm survival time (36.8 vs. 52.9 months; low vs high; $P<0.001)$ were significantly different.

3.4. Identification of Independent Prognostic Factors for HCC Prognostication. To determine the independent risk factors for HCC patient's survival, various clinicopathological factors and SNORA71A expression were analyzed in univariate and multivariate Cox regression analysis. In Tables 2 and 3, SNORA71A expression was proved to independently relate with tumor-free (HR [95\% CI]: 0.450 [0.263-0.770]; $P=$ 0.004 ) as well as long-term survival (HR [95\% CI]: 0.289 [0.127-0.657]; $P=0.003$ ) of HCC patients. These results supported that SNORA71A expression was capable of serving as the biomarker for prognosis assessment in HCC patients.

\section{Discussion}

Hepatocellular carcinoma is the most common type of liver cancer and solid tumors with fast progress and poor prognosis [16]. For HCC commonly presented with occult progression in vivo, multiple HCC patients are diagnosed at middle or even late stages [17]. Although several clinical characteristics have listed into the criteria for predicting the prognosis of HCC patients, such as tumor diameters, TNM stage, and AFP, these classification schemes are limited to become common predictors for HCC patients due to dissatisfactory sensitivity and specificity [18]. In the past 40 years, AFP has been used for monitoring the HCC, however, with poor sensitivity and specificity: $39 \%$ to $65 \%$ and $76 \%$ to $94 \%[19,20]$. Moreover, Zhang and Yang's study indicated that AFP level rarely 
TABLE 1: Correlation between SNORA71A expression and clinicopathological features in HCC patients.

\begin{tabular}{|c|c|c|c|c|}
\hline \multirow[b]{2}{*}{ Characteristics } & \multicolumn{4}{|c|}{ SNORA71A expression } \\
\hline & Total & $\begin{array}{c}\text { Low } \\
(n=66)\end{array}$ & $\begin{array}{l}\text { High } \\
(n=66)\end{array}$ & $P$ value \\
\hline Age, ys & & & & 0.138 \\
\hline$<60$ & 85 & $46(0.697)$ & $39(0.591)$ & \\
\hline$\geq 60$ & 47 & $20(0.303)$ & $27(0.409)$ & \\
\hline Gender & & & & 0.212 \\
\hline Female & 16 & $6(0.091)$ & $10(0.152)$ & \\
\hline Male & 116 & $60(0.909)$ & $56(0.848)$ & \\
\hline Hepatitis B & & & & 0.310 \\
\hline Positive & 113 & $58(0.879)$ & $11(0.167)$ & \\
\hline Negative & 19 & $8(0.121)$ & $55(0.833)$ & \\
\hline Cirrhosis & & & & 0.361 \\
\hline Present & 79 & $38(0.576)$ & $41(0.621)$ & \\
\hline Absent & 53 & $28(0.424)$ & $25(0.379)$ & \\
\hline $\operatorname{AFP}(n g / l)$ & & & & 0.429 \\
\hline$<400$ & 82 & $42(0.636)$ & $40(0.606)$ & \\
\hline$\geq 400$ & 50 & $24(0.364)$ & $26(0.394)$ & \\
\hline Tumor diameter & & & & $0.005^{*}$ \\
\hline$<5 \mathrm{~cm}$ & 34 & $10(0.152)$ & $24(0.364)$ & \\
\hline$\geq 5 \mathrm{~cm}$ & 98 & $56(0.848)$ & $42(0.636)$ & \\
\hline Multiple lesions & & & & $0.017^{*}$ \\
\hline Present & 22 & $16(0.242)$ & $6(0.091)$ & \\
\hline Absent & 110 & $50(0.758)$ & $60(0.909)$ & \\
\hline $\begin{array}{l}\text { Vessel carcinoma } \\
\text { embolus }\end{array}$ & & & & 0.160 \\
\hline Present & 34 & $20(0.303)$ & $14(0.212)$ & \\
\hline Absent & 98 & $46(0.697)$ & $52(0.788)$ & \\
\hline Microvascular invasion & & & & 0.500 \\
\hline Present & 7 & $4(0.061)$ & $3(0.045)$ & \\
\hline Absent & 125 & $62(0.939)$ & $63(0.955)$ & \\
\hline Capsular invasion & & & & $0.015^{*}$ \\
\hline Present & 49 & $31(0.470)$ & $18(0.273)$ & \\
\hline Absent & 83 & $35(0.530)$ & $48(0.727)$ & \\
\hline Differentiation & & & & $0.027^{*}$ \\
\hline Low & 70 & $41(0.621)$ & $29(0.439)$ & \\
\hline High/moderate & 62 & $25(0.379)$ & $37(0.561)$ & \\
\hline TNM stage & & & & $0.004^{*}$ \\
\hline $\mathrm{I} \sim \mathrm{II}$ & 91 & $38(0.576)$ & $53(0.803)$ & \\
\hline III IV & 41 & $28(0.424)$ & $13(0.197)$ & \\
\hline
\end{tabular}

$\mathrm{AFP}=$ alpha fetal protein; $\mathrm{TNM}=$ tumor-node-metastasis; ${ }^{*} P<0.05$; values are mean \pm standard deviation or $n(\%)$.

rose when HCC tumor mass was less than $2 \mathrm{~cm}$ in diameter [21]. Therefore, it is important and necessary to explore a novel biomarker in HCC patients.

As one kind of noncoding RNA, small nucleolar RNA (snoRNA) is originally found to make function in regulating the pseudouridine of rRNA [22]. Nowadays, emerging

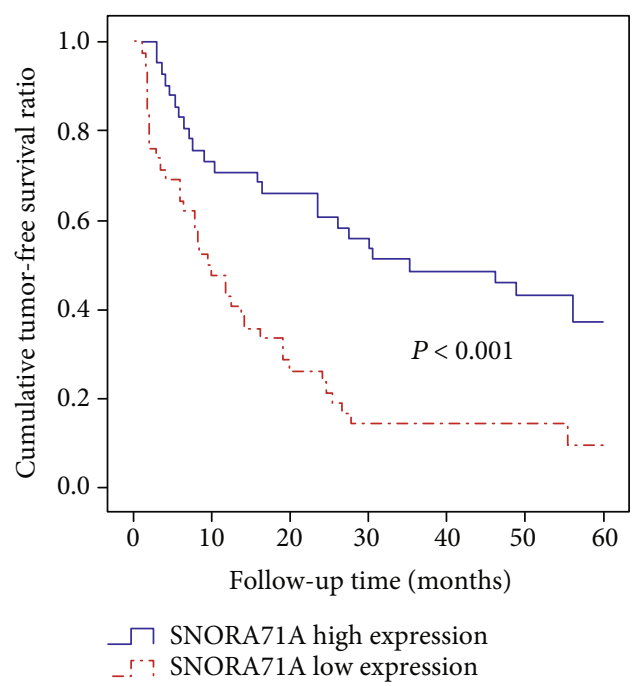

Figure 3: Cumulative tumor-free survival curves of patients in low and high SNORA71A expression subgroups.

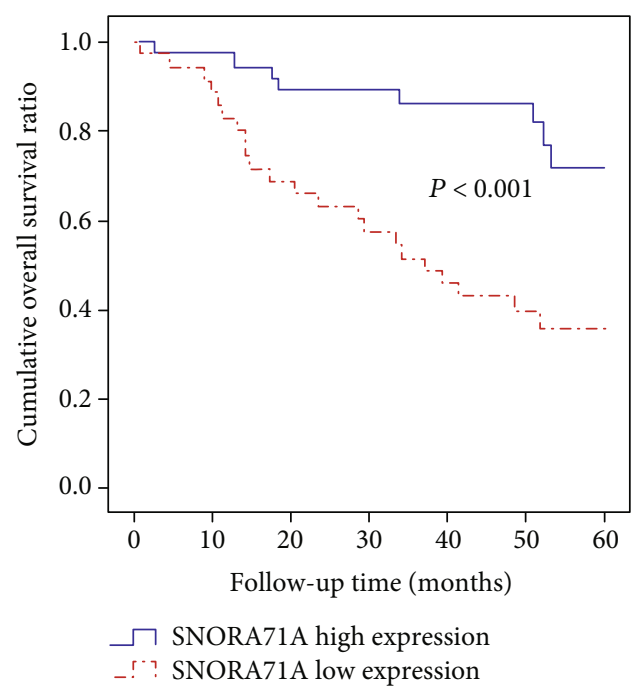

FIgURE 4: Cumulative overall survival curves of patients in low and high SNORA71A expression subgroups.

evidence showed that snoRNA also played important regulatory roles in tumorigenesis of various cancers, including liver cancer and breast cancer [23-25]. Among that, Cui et al. indicated that upregulation of snoRNA SNORA23 promoted invasion and metastasis of xenograft tumors in mice by modulating the spectrum repeat-containing nuclear envelope 2 [26]. Baral et al. reported that SNORD126, SNORD78, ACA11, SNORA47, and SNORD76 may also serve as an important prognostic marker related to clinical features. Abnormal expression of snoRNA promotes cell proliferation and leads to the development of liver cancer [27]. Besides, Cao's team reported for the first time that dysregulation of snoRNA SNORA18L5 could increase the risk of HBVassociated hepatocellular carcinoma for abnormity of ribosomal RNA maturation [28]. In HCC, moreover, snoRNA 
TABLE 2: Univariate and multivariate analysis of tumor-free survival in HCC patients.

\begin{tabular}{|c|c|c|c|c|}
\hline \multirow{2}{*}{ Clinicopathologic parameters } & \multicolumn{2}{|c|}{ Univariate analysis } & \multicolumn{2}{|c|}{ Multivariate analysis } \\
\hline & $\mathrm{HR}(95 \% \mathrm{CI})$ & $P$ & HR (95\% CI) & $P$ \\
\hline Age $(<60$ vs. $\geq 60)$ & $0.734(0.432-1.246)$ & 0.252 & & \\
\hline Gender (female vs. male) & $1.397(0.559-3.490)$ & 0.474 & & \\
\hline Hepatitis B (negative vs. positive) & $1.608(0.763-3.386)$ & 0.212 & & \\
\hline $\operatorname{AFP}(<400$ vs. $\geq 400)$ & $1.033(0.618-1.725)$ & 0.901 & & \\
\hline Cirrhosis (present vs. absent) & $1.172(0.698-1.968)$ & 0.549 & & \\
\hline Microvascular invasion (present vs. absent) & $1.272(0.460-3.520)$ & 0.643 & & \\
\hline Tumor differentiation (low vs. high/moderate) & $0.760(0.457-1.262)$ & 0.289 & & \\
\hline Capsular invasion (present vs. absent) & $1.521(0.904-2.560)$ & 0.114 & & \\
\hline Multiple lesions (present vs. absent) & $1.783(0.980-3.242)$ & 0.058 & $1.189(0.546-2.593)$ & 0.663 \\
\hline Vessel carcinoma embolus (present vs. absent) & $1.699(0.957-3.016)$ & 0.071 & $1.384(0.771-2.485)$ & 0.276 \\
\hline TNM stage (I II vs. III IV) & $2.006(1.195-3.369)$ & 0.008 & $1.577(0.922-2.699)$ & 0.097 \\
\hline Tumor diameter $(<5 \mathrm{~cm}$ vs. $\geq 5 \mathrm{~cm})$ & $2.604(1.402-4.839)$ & 0.002 & $2.154(1.140-4.070)$ & $0.018^{*}$ \\
\hline SNORA71A expression (low vs. high) & $0.385(0.228-0.650)$ & $<0.001$ & $0.450(0.263-0.770)$ & $0.004^{*}$ \\
\hline
\end{tabular}

$\mathrm{AFP}=$ alpha fetal protein; $\mathrm{TNM}=$ tumor-node-metastasis; $\mathrm{HR}=$ hazard ratio; ${ }^{*} P<0.05$ was considered statistically significant.

TABLE 3: Univariate and multivariate analysis of overall survival in HCC patients.

\begin{tabular}{|c|c|c|c|c|}
\hline \multirow{2}{*}{ Clinicopathologic parameters } & \multicolumn{2}{|c|}{ Univariate analysis } & \multicolumn{2}{|c|}{ Multivariate analysis } \\
\hline & $\mathrm{HR}(95 \% \mathrm{CI})$ & $P$ & HR $(95 \%$ CI) & $P$ \\
\hline Age (<60 vs. $\geq 60)$ & $1.067(0.514-2.216)$ & 0.861 & & \\
\hline Gender (female vs. male) & $0.541(0.231-1.266)$ & 0.157 & & \\
\hline Hepatitis B (negative vs. positive) & $0.895(0.365-2.191)$ & 0.808 & & \\
\hline AFP (<400 vs. $\geq 400)$ & $1.398(0.682-2.866)$ & 0.360 & & \\
\hline Cirrhosis (present vs. absent) & $1.208(0.575-2.540)$ & 0.618 & & \\
\hline Microvascular invasion (present vs. absent) & $1.791(0.540-5.938)$ & 0.341 & & \\
\hline Tumor differentiation (low vs. high/moderate) & $0.876(0.424-1.809)$ & 0.720 & & \\
\hline Capsular invasion (present vs. absent) & $2.175(1.063-4.452)$ & 0.033 & $1.436(0.662-3.116)$ & 0.360 \\
\hline Tumor diameter $(<5 \mathrm{~cm}$ vs. $\geq 5 \mathrm{~cm})$ & $3.480(1.210-10.005)$ & 0.021 & $1.952(0.620-6.143)$ & 0.253 \\
\hline Multiple lesions (present vs. absent) & $2.410(1.140-5.095)$ & 0.021 & $1.125(0.429-2.952)$ & 0.810 \\
\hline Vessel carcinoma embolus (present vs. absent) & $2.075(0.949-4.538)$ & 0.068 & $2.047(0.898-4.665)$ & 0.088 \\
\hline TNM stage (I II vs. III IV) & $3.061(1.480-6.331)$ & 0.003 & $2.637(1.263-5.507)$ & $0.010^{*}$ \\
\hline SNORA71A expression (low vs. high) & $0.257(0.114-0.580)$ & 0.001 & $0.289(0.127-0.657)$ & $0.003^{\prime}$ \\
\hline
\end{tabular}

$\mathrm{AFP}=$ alpha fetal protein; $\mathrm{TNM}=$ tumor-node-metastasis; $\mathrm{HR}=$ hazard ratio; ${ }^{*} P<0.05$ was considered statistically significant.

SNORD113-1 was also found to be significantly downregulated and functions as a tumor suppressor role [23]. SNHG16, SNORD76, and SnoU2_19 can regulate the development of HCC through $\mathrm{Wnt} / \beta$-catenin signaling pathway [29]. Therefore, these indicated that some specific snoRNAs had underlying abilities to serve as biomarker for clinical evaluation of HCC patients.

In our study, we for the first time analyzed the expression level of SNORA71A in both HCC cell lines and tissues and elucidated that SNORA71A was universally downregulated in HCC. Moreover, a total of 132 patients' analyses proved that SNORA71A expression was correlated with tumor diameter, multiple lesions, capsular invasion, differentiation, and TNM stage. And our results showed that SNORA71A expression was an independent risk factors for both tumor-free survival and long-term survival of HCC patients.

In conclusion, our research for the first time demonstrated that SNORA71A could serve as a novel biomarker for prognostication and therapeutic monitoring for HCC patients. And more studies are needed to explore the specific mechanisms and verify our findings in the future.
Abbreviations
HCC: Hepatocellular carcinoma
snoRNA: Small nucleolar RNA
AFP: Alpha fetal protein
TNM: Tumor-node-metastasis
HR: $\quad$ Hazard ratio. 


\section{Data Availability}

The datasets used and/or analyzed during the current study are available from the corresponding author on reasonable request.

\section{Ethical Approval}

This retrospective study was conducted according to the ethical guidelines of the 1975 Declaration of Helsinki. All participants have signed written informed consent form. Ethical approval was obtained from the Ethics Committee of the First Affiliated Hospital, School of Medicine, Zhejiang University.

\section{Conflicts of Interest}

The authors report no conflicts of interest in this work.

\section{Authors' Contributions}

W.W. and Y.D. conceived and designed the study. Y.D., Z.S., S.Z., L.Z., and Q.X. conducted the study. D.Z., H.X., Y.B., and H.D. contributed to the acquisition of data. Z.S., S.Z., C.X., and Y.G. analyzed the data. W.W., Y.D., Z.S., and S.Z. interpreted the data. W.W., Y.D., Z.S., S.Z., and L.Z. reviewed and edited the manuscript. All authors read and approved the manuscript. Yuan Ding, Zhongquan Sun, and Sitong Zhang contributed equally to this paper.

\section{Acknowledgments}

This work was supported by the National Natural Science Foundation of China (No. 81572307 and 81773096), the Major Project of Medical and Health Technology Development Program in Zhejiang Province (No. 7211902), the Key Research and Development Project of Zhejiang Province (No. 2018C03085), the Public Welfare Technology Research Project of Zhejiang Province (No. LGD19C040006), and the General Research Project of the Zhejiang Provincial Education Department (No. Y201840044).

\section{References}

[1] H. B. el-Serag and K. L. Rudolph, "Hepatocellular carcinoma: epidemiology and molecular carcinogenesis," Gastroenterology, vol. 132, no. 7, pp. 2557-2576, 2007.

[2] A. G. Singal and H. B. El-Serag, "Hepatocellular carcinoma from epidemiology to prevention: translating knowledge into practice," Clinical Gastroenterology and Hepatology, vol. 13, no. 12, pp. 2140-2151, 2015.

[3] H. E. Blum, "Hepatocellular carcinoma: therapy and prevention," World Journal of Gastroenterology, vol. 11, no. 47, pp. 7391-7400, 2005.

[4] A. M. Moon, N. S. Weiss, L. A. Beste et al., "No association between screening for hepatocellular carcinoma and reduced cancer-related mortality in patients with cirrhosis," Gastroenterology, vol. 155, no. 4, pp. 1128-1139.e6, 2018.

[5] T. Kiss, "NEW EMBO MEMBER'S REVIEW: Small nucleolar RNA-guided post-transcriptional modification of cellular
RNAs," The EMBO Journal, vol. 20, no. 14, pp. 3617-3622, 2001.

[6] Y. P. Mei, J. P. Liao, J. Shen et al., "Small nucleolar RNA 42 acts as an oncogene in lung tumorigenesis," Oncogene, vol. 31, no. 22, pp. 2794-2804, 2012.

[7] Y. Sun, E. Chen, Y. Li et al., "H/ACA box small nucleolar RNA $7 \mathrm{~B}$ acts as an oncogene and a potential prognostic biomarker in breast cancer," Cancer Cell International, vol. 19, no. 1, 2019.

[8] X.-Y. Dong, C. Rodriguez, P. Guo et al., "SnoRNA U50 is a candidate tumor-suppressor gene at $6 \mathrm{q} 14.3$ with a mutation associated with clinically significant prostate cancer," Human molecular genetics, vol. 17, no. 7, pp. 1031-1042, 2008.

[9] L. Chen, L. Han, J. Wei et al., "SNORD76, a box C/D snoRNA, acts as a tumor suppressor in glioblastoma," Scientific Reports, vol. 5, no. 1, article 8588, 2015.

[10] H. Shuwen, Y. Xi, Q. Quan, J. Yin, and D. Miao, “Can small nucleolar RNA be a novel molecular target for hepatocellular carcinoma?," Gene, vol. 733, article 144384, 2020.

[11] Y. Fu, Y. Yin, S. Peng et al., "Small nucleolar RNA host gene 1 promotes development and progression of colorectal cancer through negative regulation of miR-137," Molecular Carcinogenesis, vol. 58, no. 11, pp. 2104-2117, 2019.

[12] X. F. Liu, K. Z. Thin, X. L. Ming et al., "Small nucleolar RNA host gene 18 acts as a tumor suppressor and a diagnostic indicator in hepatocellular carcinoma," Technology in Cancer Research \& Treatment, vol. 17, 2018.

[13] P. Ganot, M. L. Bortolin, and T. Kiss, "Site-specific pseudouridine formation in preribosomal RNA is guided by small nucleolar RNAs," Cell, vol. 89, no. 5, pp. 799-809, 1997.

[14] G. Tang, Z. Zeng, W. Sun et al., "Small nucleolar RNA 71A promotes lung cancer cell proliferation, migration and invasion via MAPK/ERK pathway," Journal of Cancer, vol. 10, no. 10, pp. 2261-2275, 2019.

[15] H.-J. Schulten, M. Bangash, S. Karim et al., "Comprehensive molecular biomarker identification in breast cancer brain metastases," Journal of Translational Medicine, vol. 15, no. 1, 2017.

[16] J. D. Yang, P. Hainaut, G. J. Gores, A. Amadou, A. Plymoth, and L. R. Roberts, "A global view of hepatocellular carcinoma: trends, risk, prevention and management," Nature Reviews. Gastroenterology \& Hepatology, vol. 16, no. 10, pp. 589-604, 2019.

[17] W. Jing, P. Luo, M. Zhu, Q. Ai, H. Chai, and J. Tu, "Prognostic and diagnostic significance of sdpr-cavin-2 in hepatocellular carcinoma," Cellular Physiology and Biochemistry, vol. 39, no. 3, pp. 950-960, 2016.

[18] R. G. Gish, "Hepatocellular carcinoma: overcoming challenges in disease management," Clinical Gastroenterology and Hepatology, vol. 4, no. 3, pp. 252-261, 2006.

[19] F. Trevisani, P. E. D'Intino, A. M. Morselli-Labate et al., "Serum $\alpha$-fetoprotein for diagnosis of hepatocellular carcinoma in patients with chronic liver disease: influence of HBsAg and anti-HCV status," Journal of Hepatology, vol. 34, no. 4, pp. 570-575, 2001.

[20] M. E. Alpert, J. Uriel, and B. de Nechaud, "Alpha1 fetoglobulin in the diagnosis of human hepatoma," New England Journal of Medicine, vol. 278, no. 18, pp. 984-986, 1968.

[21] B. Zhang and B. Yang, "Combined $\alpha$ fetoprotein testing and ultrasonography as a screening test for primary liver cancer," Journal of Medical Screening, vol. 6, no. 2, pp. 108-110, 1999. 
[22] P. Ganot, M. Caizergues-Ferrer, and T. Kiss, "The family of box ACA small nucleolar RNAs is defined by an evolutionarily conserved secondary structure and ubiquitous sequence elements essential for RNA accumulation," Genes \& Development, vol. 11, no. 7, pp. 941-956, 1997.

[23] G. Xu, F. Yang, C. L. Ding et al., "Small nucleolar RNA 113-1 suppresses tumorigenesis in hepatocellular carcinoma," Molecular Cancer, vol. 13, no. 1, 2014.

[24] H. Su, T. Xu, S. Ganapathy et al., "Elevated snoRNA biogenesis is essential in breast cancer," Oncogene, vol. 33, no. 11, pp. 1348-1358, 2014.

[25] Z. Siprashvili, D. E. Webster, D. Johnston et al., "The noncoding RNAs SNORD50A and SNORD50B bind K-Ras and are recurrently deleted in human cancer," Nature Genetics, vol. 48, no. 1, pp. 53-58, 2015.

[26] L. Cui, K. Nakano, S. Obchoei et al., "Small nucleolar noncoding RNA SNORA23, up-regulated in human pancreatic ductal adenocarcinoma, regulates expression of spectrin repeatcontaining nuclear envelope 2 to promote growth and metastasis of xenograft tumors in mice," Gastroenterology, vol. 153, no. 1, pp. 292-306.e2, 2017.

[27] D. Baral, L. Wu, G. Katwal, X. Yan, Y. Wang, and Q. Ye, "Clinical significance and biological roles of small nucleolar RNAs in hepatocellular carcinoma (Review)," Biomedical reports, vol. 8, no. 4, pp. 319-324, 2018.

[28] P. Cao, A. Yang, R. Wang et al., "Germline duplication of SNORA18L5 increases risk for HBV-related hepatocellular carcinoma by altering localization of ribosomal proteins and decreasing levels of p53," Gastroenterology, vol. 155, no. 2, pp. 542-556, 2018.

[29] Y. Shuai, Z. Ma, J. Lu, and J. Feng, "LncRNA SNHG15: a new budding star in human cancers," Cell Proliferation, vol. 53, no. 1 , article e12716, 2020. 